\title{
Decomposing complete multipartite graphs into closed trails of arbitrary even lengths
}

\author{
Benjamin R. Smith, Selda Küçükçifçi and Emine Şule Yazıcı \\ Department of Mathematics, Koç University \\ Rumelifeneri Yolu, 34450, Sarıer, Istanbul, TURKEY
}

\begin{abstract}
We prove that any complete multipartite graph with parts of even size can be decomposed into closed trails with prescribed even lengths.
\end{abstract}

\section{Introduction}

All graphs considered in this paper are simple. We denote the vertex set and edge set of a graph $G$ by $V(G)$ and $E(G)$ respectively. Furthermore, we define the size of $G$ to be $|E(G)|$.

A complete multipartite graph $K_{a_{1}, a_{2}, \ldots, a_{n}}$ has $\sum_{i=1}^{n} a_{i}$ vertices, partitioned into $n$ parts of sizes $a_{1}, a_{2}, \ldots, a_{n}$, with two vertices adjacent if and only if they belong to different parts. We note that a (nontrivial) complete multipartite graph is even - that is, each of its vertices has even degree - if and only if either $a_{1}, a_{2}, \ldots, a_{n}$ are all even, or $n$ is odd and $a_{1}, a_{2}, \ldots, a_{n}$ are all odd.

A sequence $v_{0} e_{1} v_{1} e_{2} v_{2} \ldots v_{l-1} e_{l} v_{l}$, where $v_{i}$ is a vertex for each $i \in\{0,1, \ldots, l\}$ and $e_{j}$ is an edge with end vertices $v_{j-1}$ and $v_{j}$ for each $j \in\{1,2, \ldots, l\}$, is a trail of length $l$ if the edges $e_{1}, e_{2}, \ldots, e_{l}$ are distinct. We denote such a trail by $\left[v_{0}, v_{1}, \ldots, v_{l}\right]$ and say it is closed if $v_{0}=v_{l}$ and open otherwise. We will also use the terms closed trail and open trail 
to refer to graphs whose vertices and edges may be ordered to form such sequences. That is, a closed trail of length $l$ may be regarded as a connected even graph of size $l$, and an open trail of length $l$ may be regarded as a connected graph of size $l$ with precisely two vertices of odd degree. Hence the notation $\left[v_{0}, v_{1}, \ldots, v_{l}\right]$ may also be taken to mean the graph with vertex set $\left\{v_{0}, v_{1}, \ldots, v_{l}\right\}$ and edge set $\left\{v_{0} v_{1}, v_{1} v_{2}, \ldots, v_{l-1} v_{l}\right\}$. Any trail in a graph $G$ which contains all the edges of $G$ is called an Euler trail. Thus $G$ contains an Euler trail if and only if it is connected and is either even, or contains precisely two vertices of odd degree.

If $H$ is a subgraph of $G$, we write $G \backslash H$ to denote the graph obtained by removing the edges of $H$ from $G$ and deleting any isolated vertices. Conversely, if $G$ and $H$ are edge-disjoint, we write $G \cup H$ to denote the graph obtained by adding the edges, and vertices if necessary, of $H$ to $G$. We say there is a decomposition of $G$ into $H_{1}, H_{2}, \ldots, H_{t}$, if $H_{1}, H_{2}, \ldots, H_{t}$ are pairwise edge-disjoint subgraphs of $G$ such that $E(G)=E\left(H_{1}\right) \cup E\left(H_{2}\right) \cup \cdots \cup E\left(H_{t}\right)$; that is, if $G=H_{1} \cup H_{2} \cup \cdots \cup H_{t}$.

A list $\left(l_{1}, l_{2}, \ldots, l_{t}\right)$ of positive integers is said to be $G$-admissible if $\sum_{i=1}^{t} l_{i}=|E(G)|$. We let $\operatorname{Lct}(G)$ denote the set of all positive integers $l$ for which $G$ contains a closed trail of length $l$. Then a connected even graph $G$ is said to be arbitrarily decomposable into closed trails (or ADCT for short), if there is a decomposition of $G$ into $t$ closed trails of lengths $l_{1}, l_{2}, \ldots, l_{t}$, for every $G$-admissible list $\left(l_{1}, l_{2}, \ldots, l_{t}\right)$ in which each $l_{i} \in \operatorname{Lct}(G)$. Necessary and sufficient conditions for a graph $G$ to be ADCT are not known in general. However, numerous results have been obtained for cases where $G$ is a complete multipartite graph, with certain restrictions placed on either the size or the number of its parts. For example, Balister [1] showed that $K_{a_{1}, a_{2}, \ldots, a_{n}}$ is ADCT whenever either each $a_{i}=1$ and $n$ is (necessarily) odd (hence $K_{a_{1}, a_{2}, \ldots, a_{n}}$ is a complete graph of odd order), or each $a_{i}=2$ (hence $K_{a_{1}, a_{2}, \ldots, a_{n}}$ is a complete graph of even order minus a 1-factor). In [4] Horňák and Woźniak showed that $K_{a_{1}, a_{2}}$ is $\mathrm{ADCT}$ whenever (necessarily) both $a_{1}$ and $a_{2}$ are even, and in [2] Billington and Cavenagh showed that $K_{a_{1}, a_{2}, a_{3}}$ is ADCT if and only if either $a_{1}=a_{2}=a_{3}$, or $\left\{a_{1}, a_{2}, a_{3}\right\} \in\{\{1,1,3\},\{1,1,5\}\}$. In [2] Billington and Cavenagh also conjectured that $K_{a_{1}, a_{2}, \ldots, a_{n}}$ is ADCT whenever $a_{1}=a_{2}=\cdots=a_{n}=a$ say, and (necessarily) either $n$ is odd 
or $a$ is even - the so-called "equipartite case". Progress toward a proof of this conjecture for $n \geq 4$ has so far been limited to considering decompositions of complete equipartite graphs in which each of the closed trails is of the same length; that is, where $l_{1}=l_{2}=\cdots=l_{t}$ (see the independent results of Burgess and Šajna [3] and Smith [5]).

Instead of focusing on the equipartite case, in this paper we examine the case where each part of $K_{a_{1}, a_{2}, \ldots, a_{n}}$ is of even size. The tripartite case results of Billington and Cavenagh [2] make it clear that in general, if $a_{1}, a_{2}, \ldots, a_{n}$ are not all equal, then $K_{a_{1}, a_{2}, \ldots, a_{n}}$ may not be ADCT. However, we show that if one considers only decompositions into closed trails of even lengths, then some general results can be obtained. With this in mind we introduce the following new terminology. Let Lcet $(G)$ denote the set of all positive even integers $l$ for which $G$ contains a closed trail of length $l$. Then a connected even graph $G$ is said to be arbitrarily decomposable into closed even trails (or ADCET for short), if there is a decomposition of $G$ into $t$ closed trails of lengths $l_{1}, l_{2}, \ldots, l_{t}$, for every $G$-admissible list $\left(l_{1}, l_{2}, \ldots, l_{t}\right)$ in which each $l_{i} \in \operatorname{Lcet}(G)$. Our main result is then as follows.

Theorem 1.1 If $a_{1}, a_{2}, \ldots, a_{n}$ are positive integers and $n \geq 2$, then the graph $K_{2 a_{1}, 2 a_{2}, \ldots, 2 a_{n}}$ is ADCET.

This effectively generalizes the results of Horňák and Woźniak [4] who proved the above theorem in the specific case $n=2$. (We note that $K_{2 a_{1}, 2 a_{2}}$ is bipartite and hence $\operatorname{Lct}\left(K_{2 a_{1}, 2 a_{2}}\right)=$ $\operatorname{Lcet}\left(K_{2 a_{1}, 2 a_{2}}\right)$, and ADCT and ADCET are equivalent in this case.) Our result is obtained using a different method to that employed by Horňák and Woźniak and thus also provides an alternative (simpler) proof of the bipartite case.

\section{Stars and non-stars}

In this section we present some useful preliminary results. We begin with some definitions.

The $k$-star $S_{k}$ is a graph consisting of $k$ edges which share a common vertex, called the center vertex of the star. We refer to the set of $k$ vertices in $V\left(S_{k}\right)$ which are not the center as the boundary of the star. A graph $G$ is said to be a non-star if it is connected and is 
not isomorphic to $S_{k}$ for any $k \geq 3$. Note that the unique (up to isomorphism) connected graphs of sizes 1 and 2 are in fact both stars and non-stars according to our definitions. The graph $G$ is said to be arbitrarily decomposable into non-stars (or ADNS for short), if there is a decomposition of $G$ into $t$ non-stars of sizes $l_{1}, l_{2}, \ldots, l_{t}$, for every $G$-admissible list $\left(l_{1}, l_{2}, \ldots, l_{t}\right)$. We note that since trails are non-stars, it is easy to see that $G$ is ADNS whenever it contains an Euler trail (as a suitable decomposition can always be obtained by simply "chopping" the Euler trail into smaller trails of the required sizes).

We define $G(2)$ to be the graph obtained from $G$ by replacing each vertex $v$ with the pair of vertices $v^{(1)}$ and $v^{(2)}$, and replacing each edge $u v$ with the edges $u^{(1)} v^{(1)}, u^{(1)} v^{(2)}, u^{(2)} v^{(1)}$ and $u^{(2)} v^{(2)}$. Hence $G(2)$ is four times the size of $G$.

Lemma 2.1 Suppose $l_{1}$ and $l_{2}$ are odd integers with $l_{1}, l_{2} \geq 3$, and $H$ is a non-star of size $\left(l_{1}+l_{2}\right) / 2$. Then there exists a decomposition of $H(2)$ into two closed trails of lengths $2 l_{1}$ and $2 l_{2}$.

Proof Let $k=\left(l_{1}+l_{2}\right) / 2 \geq 3$ and $H$ be a non-star of size $k$. We prove the result by induction on $k$.

Suppose $k=3$. Hence $l_{1}=l_{2}=3$ and $H$ contains an Euler trail, say $[u, v, w, x]$ (note that $u$ and $x$ need not be distinct). A suitable decomposition of $H(2)$ is thus given by the closed trails $\left[u^{(1)}, v^{(1)}, w^{(1)}, x^{(1)}, w^{(2)}, v^{(2)}, u^{(1)}\right]$ and $\left[u^{(2)}, v^{(1)}, w^{(2)}, x^{(2)}, w^{(1)}, v^{(2)}, u^{(2)}\right]$.

Suppose now that the result holds for $k=i \geq 3$. We prove the result also holds for $k=i+1$. Let $l_{1}$ and $l_{2}$ be odd integers, $l_{1}, l_{2} \geq 3$, such that $k=i+1=\left(l_{1}+l_{2}\right) / 2$. Since $i+1 \geq 4$, we may assume $l_{1} \geq 5$. We note that for at least one edge of $H$, say $x y$, the subgraph of $H$, say $H^{\prime}$, obtained by removing the edge $x y$ from $H$ and deleting any isolated vertices, is a non-star of size $i \geq 3$. Since $i=\left(\left(l_{1}-2\right)+l_{2}\right) / 2$ with $l_{1}-2$, $l_{2}$ odd and $l_{1}-2, l_{2} \geq 3$, by our assumption we have that there exists a decomposition of $H^{\prime}(2)$ into closed trails, say $T_{1}^{\prime}$ and $T_{2}^{\prime}$, of lengths $2 l_{1}-4$ and $2 l_{2}$, respectively. Furthermore, if at least one of the vertices $x^{(1)}, x^{(2)}, y^{(1)}$ or $y^{(2)}$ belongs to $V\left(T_{1}^{\prime}\right)$, then the result follows by letting $T_{1}=T_{1}^{\prime} \cup\left[x^{(1)}, y^{(1)}, x^{(2)}, y^{(2)}, x^{(1)}\right]$ and $T_{2}=T_{2}^{\prime}$. Suppose then that 
$\left\{x^{(1)}, x^{(2)}, y^{(1)}, y^{(2)}\right\} \cap V\left(T_{1}^{\prime}\right)=\emptyset$. Let $P$ be a shortest path in $H^{\prime}(2)$ connecting a vertex in $\left\{x^{(1)}, x^{(2)}, y^{(1)}, y^{(2)}\right\} \cap V\left(T_{2}^{\prime}\right)$ with a vertex in $V\left(T_{1}^{\prime}\right)$. Such a path must exist since $H^{\prime}$ is connected and contains at least one of the vertices $x$ or $y$. Without loss of generality we may assume that $P=\left[v_{1}^{(1)}, v_{2}^{(1)}, \ldots, v_{n}^{(1)}\right]$ where $v_{1}=y, n \geq 2$ and $v_{1}, \ldots, v_{n}$ are (distinct) vertices in $V\left(H^{\prime}\right)$, with each $v_{i} \neq x$. Let $v_{0}=x, T_{1}=T_{1}^{\prime} \cup\left[v_{n}^{(1)}, v_{n-1}^{(1)}, v_{n-2}^{(1)}, v_{n-1}^{(2)}, v_{n}^{(1)}\right]$ and $T_{2}=\left(T_{2}^{\prime} \cup\left[x^{(1)}, y^{(1)}, x^{(2)}, y^{(2)}, x^{(1)}\right]\right) \backslash\left[v_{n}^{(1)}, v_{n-1}^{(1)}, v_{n-2}^{(1)}, v_{n-1}^{(2)}, v_{n}^{(1)}\right]$. The result then follows as long as both $T_{1}$ and $T_{2}$ are connected. It is clear that $T_{1}$ is connected. Furthermore, $T_{2}$ is connected except in the case that it contains exactly two nontrivial connected components, say $C_{1}$ and $C_{2}$, with $v_{n}^{(1)} \in V\left(C_{1}\right)$ and $v_{n}^{(2)} \in V\left(C_{2}\right)$. Assume this is the case. Let $a$ and $b$ be distinct vertices such that $C_{1}$ contains the (open) trail $\left[a, v_{n}^{(1)}, b\right]$. Thus $T_{1}$ contains the (open) trail $\left[a, v_{n}^{(2)}, b\right]$. We switch the edges $a v_{n}^{(1)}$ and $v_{n-1}^{(1)} v_{n}^{(2)}$ from $T_{2}$ to $T_{1}$ and switch the edges $a v_{n}^{(2)}$ and $v_{n-1}^{(1)} v_{n}^{(1)}$ from $T_{1}$ to $T_{2}$. It is easy to see that these modified $T_{1}$ and $T_{2}$ are closed trails of the required lengths. The result follows.

The main ingredient in our proof of Theorem 1.1 is the following lemma.

Lemma 2.2 If $G$ is ADNS, then $G(2)$ is ADCET.

Proof Let $\left(2 l_{1}, 2 l_{2}, \ldots, 2 l_{t}\right)$ be a $G(2)$-admissible list in which each $2 l_{i} \in \operatorname{Lcet}(G(2))$. Note that $l_{i}>1$ for all $i$. Suppose first that each $l_{i}$ is even. Then $\left(l_{1} / 2, l_{2} / 2, \ldots, l_{t} / 2\right)$ is a $G$ admissible list. Since $G$ is ADNS we have that $G$ decomposes into non-stars $H_{1}, H_{2}, \ldots, H_{t}$ of sizes $l_{1} / 2, l_{2} / 2, \ldots, l_{t} / 2$. Thus the graphs $H_{1}(2), H_{2}(2), \ldots, H_{t}(2)$ are closed trails of lengths $2 l_{1}, 2 l_{2}, \ldots, 2 l_{t}$, which together decompose $G(2)$ as required.

Suppose then that there is at least one $l_{i}$ which is odd. Note that since the size of $G(2)$ is a multiple of 4 there must be an even number of $l_{i}$ which are odd. Thus without loss of generality we may assume the values $l_{1}, l_{2}, \ldots, l_{2 t^{\prime}}$ are odd and that either $t=2 t^{\prime}$ or $t>2 t^{\prime}$ and the values $l_{2 t^{\prime}+1}, \ldots, l_{t}$ are even. Then $\left(\left(l_{1}+l_{2}\right) / 2,\left(l_{3}+l_{4}\right) / 2, \ldots,\left(l_{2 t^{\prime}-1}+l_{2 t^{\prime}}\right) / 2\right)$ (or $\left(\left(l_{1}+l_{2}\right) / 2,\left(l_{3}+l_{4}\right) / 2, \ldots,\left(l_{2 t^{\prime}-1}+l_{2 t^{\prime}}\right) / 2, l_{2 t^{\prime}+1} / 2, \ldots, l_{t} / 2\right)$ if $\left.t>2 t^{\prime}\right)$ is a $G$-admissible list. Since $G$ is ADNS we have that $G$ decomposes into non-stars $H_{1}, H_{2}, \ldots, H_{t-t^{\prime}}$ with 
$\left|E\left(H_{i}\right)\right|=\left(l_{2 i-1}+l_{2 i}\right) / 2$ if $i \in\left\{1,2, \ldots, t^{\prime}\right\}$, and $\left|E\left(H_{i}\right)\right|=l_{i+t^{\prime}} / 2$ if $t>2 t^{\prime}$ and $i \in$ $\left\{1+t^{\prime}, \ldots, t-t^{\prime}\right\}$. Thus the graphs $H_{1}(2), H_{2}(2), \ldots, H_{t-t^{\prime}}(2)$ are closed trails of lengths $4\left|E\left(H_{1}\right)\right|, 4\left|E\left(H_{2}\right)\right|, \ldots, 4\left|E\left(H_{t-t^{\prime}}\right)\right|$, which together decompose $G(2)$. The result then follows by noting that for each $i \in\left\{1,2, \ldots, t^{\prime}\right\}$ the graph $H_{i}(2)$ can be further decomposed into two closed trails of lengths $2 l_{2 i-1}$ and $2 l_{2 i}$ by Lemma 2.1 .

It is clear from Lemma 2.2 that, since $K_{2 a_{1}, 2 a_{2}, \ldots, 2 a_{n}} \cong K_{a_{1}, a_{2}, \ldots, a_{n}}(2)$, for $n \geq 2$, Theorem 1.1 holds for particular values of $a_{1}, a_{2}, \ldots, a_{n}$ if $K_{a_{1}, a_{2}, \ldots, a_{n}}$ is ADNS. With this in mind we now show that $K_{a_{1}, a_{2}, \ldots, a_{n}}$ is almost always ADNS. In order to do this we use the following lemma.

Lemma 2.3 Suppose $G$ is connected and has a subgraph $G^{\prime}$ such that

- $G \backslash G^{\prime}$ contains an Euler trail; and

- each connected component of $G^{\prime}$ is a star whose boundary is a subset of $V\left(G \backslash G^{\prime}\right)$.

Then $G$ has a decomposition into $t$ non-stars of sizes $l_{1}, l_{2}, \ldots, l_{t}$, for any $G$-admissible list $\left(l_{1}, l_{2}, \ldots, l_{t}\right)$ in which each $l_{i} \neq 3$.

Proof Our aim is to order the edges of $G$ in such a way that each pair of consecutive edges shares a vertex, and at most three consecutive edges share the same vertex. Hence no four consecutive edges form a star. Since $l_{i} \neq 3$ for all $i$, it is then clear that suitable non-stars can then be obtained by simply letting the "first" $l_{1}$ edges form the edge set of one such graph, the next $l_{2}$ edges form the edge set of another, and so on.

Suppose $\left[u_{0}, u_{1}, \ldots, u_{n}\right]$ is an Euler trail of $G \backslash G^{\prime}$ (hence the vertices $u_{i}$ need not be distinct). For each $i \in\{1,2, \ldots, n\}$ let $e_{i}$ be the edge $u_{i-1} u_{i}$. Note that in the ordering $e_{1} e_{2}, \ldots e_{n}$ of the edges of $G \backslash G^{\prime}$, each pair of consecutive edges shares a vertex and at most two consecutive edges share the same vertex. We then obtain our desired ordering of the edges of $G$ by modifying this ordering as follows. Suppose $\left|E\left(G^{\prime}\right)\right|=k$ and $\left\{v_{1}, v_{2}, \ldots, v_{k}\right\}$ is the union of the boundaries of each component star in $G^{\prime}$. By assumption we have that each 
$v_{i} \in V\left(G \backslash G^{\prime}\right)$. For each $i \in\{1,2, \ldots, k\}$ we let $f_{i}$ be the (unique) edge in $E\left(G^{\prime}\right)$ which is incident with the vertex $v_{i}$, and we define $i^{\prime}$ to be the least value in $\{0,1, \ldots, n\}$ such that $v_{i}=u_{i^{\prime}}$. Then, for each $i \in\{1,2, \ldots, k\}$, we insert the edge $f_{i}$ into the above order $e_{1} e_{2} \ldots e_{n}$ immediately after the edge $e_{i^{\prime}}$ (or immediately preceding the edge $e_{i^{\prime}+1}$ in the case $i^{\prime}=0$ ). It is clear that this modified ordering has the required properties.

Theorem 2.4 If $a_{1}, a_{2}, \ldots, a_{n}$ are positive integers and $n \geq 2$, then the graph $K_{a_{1}, a_{2}, \ldots, a_{n}}$ is ADNS if and only if either $n \geq 3$, or $n=2$ and $\left\{a_{1}, a_{2}\right\} \neq\{1, a\}$ for each $a \geq 3$.

Proof Suppose first that $n=2$ and $\left\{a_{1}, a_{2}\right\}=\{1, a\}$ for some $a \geq 1$. Then $K_{a_{1}, a_{2}}$ is itself a star and hence is clearly ADNS if and only if $a \in\{1,2\}$. Suppose then that either $n \geq 3$, or $n=2$ and $a_{1}, a_{2} \geq 2$. It remains to prove that $K_{a_{1}, a_{2}, \ldots, a_{n}}$ is ADNS.

Let $x$ be the number of $a_{i}$ which are odd and $y$ be the number of $a_{i}$ which are even. Hence $x+y=n$. If $x>0$, let $V_{1}, V_{2}, \ldots, V_{x}$ be the partite sets of $K_{a_{1}, a_{2}, \ldots, a_{n}}$ having odd size and let $v=\sum_{i=1}^{x}\left|V_{i}\right|$, with $v=0$ if $x=0$. Similarly, if $y>0$, let $W_{1}, W_{2}, \ldots, W_{y}$ be the partite sets of $K_{a_{1}, a_{2}, \ldots, a_{n}}$ having even size and let $w=\sum_{i=1}^{y}\left|W_{i}\right|$, with $w=0$ if $y=0$. Without loss of generality we may assume that $\left|V_{1}\right| \leq\left|V_{2}\right| \leq \cdots \leq\left|V_{x}\right|$ and $\left|W_{1}\right| \leq\left|W_{2}\right| \leq \cdots \leq\left|W_{y}\right|$. If either $v \in\{0,2\}$, or $w=0$ and $v$ is odd, then $K_{a_{1}, a_{2}, \ldots, a_{n}}$ has an Euler trail and the result follows. Thus we may assume that if $v$ is even, then $v \geq 4$, and if $v$ is odd, then $w>0$.

Let $\left(l_{1}, l_{2}, \ldots, l_{t}\right)$ be an arbitrary $\left(K_{a_{1}, a_{2}, \ldots, a_{n}}\right)$-admissible sequence and let $\alpha$ be the number of times 3 occurs in the list. We first deal with the case in which $\alpha=0$. If $\alpha=0$ and $v$ is odd, we let $v_{1} \in V_{1}$. Then $G=K_{a_{1}, a_{2}, \ldots, a_{n}}$ satisfies the conditions of Lemma 2.3, with $G^{\prime}$ a star having center vertex $v_{1}$ and boundary $\bigcup_{i=1}^{y} W_{i}$. Similarly if $\alpha=0$ and $v$ is even, we let $k=\left|V_{1}\right|, V_{1}=\left\{v_{1}, v_{2}, \ldots, v_{k}\right\}$ and $u_{1}, u_{2}, \ldots, u_{k}$ be distinct vertices in $V_{2}$. Then $G=K_{a_{1}, a_{2}, \ldots, a_{n}}$ satisfies the conditions of Lemma 2.3, with $G^{\prime}$ either the star having center vertex $v_{1}$ and boundary $\bigcup_{i=2}^{x} V_{i}$ (in the case $k=1$ ), or $G^{\prime}$ the union of the star having center vertex $v_{1}$ and boundary $\left(\bigcup_{i=2}^{x} V_{i}\right) \backslash\left\{u_{1}, u_{2}\right\}$ and the star having center vertex $u_{1}$ and boundary $\left\{v_{2}, v_{3}, \ldots, v_{k}\right\}$ (in the case $k \geq 3$ ). 
Thus it remains to deal with the cases in which $\alpha \geq 1$. Without loss of generality we may assume that $l_{1}=l_{2}=\cdots=l_{\alpha}=3$. Our aim is to show that for some positive $\alpha^{\prime} \leq \alpha$ we can decompose $K_{a_{1}, a_{2}, \ldots, a_{n}}$ into two subgraphs, say $G$ and $H$, in such a way that $H$ has a decomposition into $\alpha^{\prime}$ non-stars of size 3, and either $G$ has an Euler trail or $\alpha^{\prime}=\alpha$ and $G$ satisfies the conditions of Lemma 2.3. (The result then follows since in either case the graph $G$ has a decomposition into $t-\alpha^{\prime}$ non-stars of sizes $l_{\alpha^{\prime}+1}, l_{\alpha^{\prime}+2}, \ldots, l_{t}$.) We now split the problem according to the parity of $v$.

\section{Case 1: $v$ is odd.}

Subcase 1.1: $v \geq 3$

Let $v_{1}, v_{2}$ and $v_{3}$ be distinct vertices in $\bigcup_{i=1}^{x} V_{i}$ and partition the vertices in $\bigcup_{i=1}^{y} W_{i}$ into pairs, say $P_{1}=\left\{w_{1}, w_{2}\right\}, P_{2}, \ldots, P_{w / 2}$. For each $i \in\{1,2, \ldots, w / 2\}$ we let $H_{i}$ be the complete bipartite graph with partite sets $\left\{v_{1}, v_{2}, v_{3}\right\}$ and $P_{i}$. It is clear that each $H_{i}$ can be decomposed into two non-stars of size 3. Then, if $\alpha \geq w$, we let $H=\bigcup_{i=1}^{w / 2} H_{i}$. Observe that the degrees of all vertices in $G=K_{a_{1}, a_{2}, \ldots, a_{n}} \backslash H$ are even, and thus $G$ has a (closed) Euler trail. Similarly, if $\alpha<w$ and $\alpha$ is even, we let $H=\bigcup_{i=1}^{\alpha / 2} H_{i}$ and hence $G=K_{a_{1}, a_{2}, \ldots, a_{n}} \backslash H$ satisfies the conditions of Lemma 2.3, with $G^{\prime}$ the star having center vertex $v_{1}$ and boundary $\bigcup_{i=\alpha / 2+1}^{w / 2} P_{i}$. Finally, if $\alpha<w$ and $\alpha$ is odd, we let $H=\left(\bigcup_{i=2}^{(\alpha+1) / 2} H_{i}\right) \cup\left[w_{1}, v_{1}, w_{2}, v_{2}\right]$. Then $G=K_{a_{1}, a_{2}, \ldots, a_{n}} \backslash H$ either contains an (open) Euler trail (in the case $\alpha=w-1$ ) or satisfies the conditions of Lemma 2.3 , with $G^{\prime}$ the star having center vertex $v_{1}$ and boundary $\bigcup_{i=(\alpha+3) / 2}^{w / 2} P_{i}$ (in the case $\left.\alpha<w-1\right)$.

Subcase 1.2: $v=1$

Note that in this case $y \geq 2$. Let $V_{1}=\left\{v_{1}\right\}, w_{1}$ and $w_{2}$ be distinct vertices in $W_{1}$, and $u_{1}$ and $u_{2}$ be distinct vertices in $W_{y}$. Partition the vertices in $\bigcup_{i=2}^{y} W_{i}$ into pairs, say $P_{0}=\left\{u_{1}, u_{2}\right\}, P_{1}, \ldots, P_{r}$, and partition the vertices in $W_{1}$ into pairs, say $P_{r+1}=$ $\left\{w_{1}, w_{2}\right\}, P_{r+2}, \ldots, P_{(w-2) / 2}=\left\{w_{1}^{\prime}, w_{2}^{\prime}\right\}$ (note that if $\left|W_{1}\right|=2$, then $\left.P_{r+1}=P_{(w-2) / 2}\right)$. For each $i \in\{r+1, r+2, \ldots,(w-2) / 2\}$ we let $H_{i}$ be the complete bipartite graph with partite sets $\left\{v_{1}, u_{1}, u_{2}\right\}$ and $P_{i}$, and if $r>0$, for each $i \in\{1,2, \ldots, r\}$, we let $H_{i}$ be the complete bipartite graph with partite sets $\left\{v_{1}, w_{1}, w_{2}\right\}$ and $P_{i}$. It is clear that each $H_{i}$ can be decom- 
posed into two non-stars of size 3. Then, if $\alpha \geq w-2$, we let $H=\bigcup_{i=1}^{(w-2) / 2} H_{i}$ and thus $G=K_{a_{1}, a_{2}, \ldots, a_{n}} \backslash H$ has an (open) Euler trail. Similarly, if $\alpha<w-2$ and $\alpha$ is even, we let $H=\bigcup_{i=1}^{\alpha / 2} H_{i}$ and hence $G=K_{a_{1}, a_{2}, \ldots, a_{n}} \backslash H$ satisfies the conditions of Lemma 2.3, with $G^{\prime}$ the star having center vertex $v_{1}$ and boundary $\bigcup_{i=\alpha / 2+1}^{(w-2) / 2} P_{i}$. Finally, if $\alpha<w-2$ and $\alpha$ is odd, we let $H=\left(\bigcup_{i=1}^{(\alpha-1) / 2} H_{i}\right) \cup\left[w_{1}^{\prime}, u_{1}, w_{2}^{\prime}, u_{2}\right]$. Then $G=K_{a_{1}, a_{2}, \ldots, a_{n}} \backslash H$ either contains an (open) Euler trail (in the case $\alpha=w-3$ ) or satisfies the conditions of Lemma 2.3, with $G^{\prime}$ the star having center vertex $v_{1}$ and boundary $\bigcup_{i=(\alpha+1) / 2}^{(w-4) / 2} P_{i}$ (in the case $\alpha<w-3$ ).

Case 2: $v$ is even.

Recall that $v \geq 4$ in this case.

Subcase 2.1: $\left|V_{1}\right| \geq 3$

Let $v_{1}, v_{2}$ and $v_{3}$ be distinct vertices in $V_{1}$, and let $w_{1}, w_{2}$ and $w_{3}$ be distinct vertices in $V_{x}$. Partition the vertices in $\left(\bigcup_{i=2}^{x} V_{i}\right) \backslash\left\{w_{1}\right\}$ into pairs, say $P_{0}=\left\{w_{2}, w_{3}\right\}, P_{1}, \ldots, P_{r}$, and partition the vertices in $V_{1} \backslash\left\{v_{1}\right\}$ into pairs, say $P_{r+1}, P_{r+2}, \ldots, P_{(v-4) / 2}=\left\{v_{2}, v_{3}\right\}$. For each $i \in\{r+1, r+2, \ldots,(v-4) / 2\}$ we let $H_{i}$ be the complete bipartite graph with partite sets $\left\{w_{1}, w_{2}, w_{3}\right\}$ and $P_{i}$, and if $r>0$, for each $i \in\{1,2, \ldots, r\}$, we let $H_{i}$ be the complete bipartite graph with partite sets $\left\{v_{1}, v_{2}, v_{3}\right\}$ and $P_{i}$. It is clear that each $H_{i}$ can be decomposed into two non-stars of size 3. If $\alpha \geq v-4$, we let $H=\left(\bigcup_{i=1}^{(v-6) / 2} H_{i}\right) \cup\left[v_{1}, w_{2}, v_{2}, w_{1}\right] \cup\left[v_{2}, w_{3}, v_{3}, w_{2}\right]$ and thus $G=K_{a_{1}, a_{2}, \ldots, a_{n}} \backslash H$ has an (open) Euler trail. Similarly, if $\alpha<v-4$ and $\alpha$ is even, we let $H=\bigcup_{i=1}^{\alpha / 2} H_{i}$ and hence $G=K_{a_{1}, a_{2}, \ldots, a_{n}} \backslash H$ satisfies the conditions of Lemma 2.3, with $G^{\prime}$ either the star having center vertex $w_{1}$ and boundary $\left\{v_{1}\right\} \cup \bigcup_{i=\alpha / 2+1}^{(v-4) / 2} P_{i}$ (in the case $\alpha / 2+1>r$ ), or $G^{\prime}$ the union of the star having center vertex $v_{1}$ and boundary $\left(\bigcup_{i=\alpha / 2+1}^{r} P_{i}\right) \cup\left\{w_{3}\right\}$ and the star having center vertex $w_{1}$ and boundary $\bigcup_{i=r+1}^{(v-4) / 2} P_{i}$ (in the case $\alpha / 2+1 \leq r)$. Finally, if $\alpha<v-4$ and $\alpha$ is odd, we let $H=\left(\bigcup_{i=1}^{(\alpha-1) / 2} H_{i}\right) \cup\left[v_{1}, w_{1}, v_{2}, w_{2}\right]$. Then $G=K_{a_{1}, a_{2}, \ldots, a_{n}} \backslash H$ satisfies the conditions of Lemma 2.3, with $G^{\prime}$ either the star having center vertex $w_{1}$ and boundary $\left(\bigcup_{i=(\alpha+1) / 2}^{(v-4) / 2} P_{i}\right) \backslash\left\{v_{2}\right\}$ (in the case $(\alpha+1) / 2>r$ ), or $G^{\prime}$ the union of the star having center vertex $v_{1}$ and boundary $\bigcup_{i=(\alpha+1) / 2}^{r} P_{i}$ and the star having center vertex $w_{1}$ and boundary $\left(\bigcup_{i=r+1}^{(v-4) / 2} P_{i}\right) \backslash\left\{v_{2}\right\}$ (in the case $\left.(\alpha+1) / 2 \leq r\right)$. 


\section{Subcase 2.2: $\left|V_{1}\right|=1$}

Let $V_{1}=\left\{v_{1}\right\}$.

Subcase 2.2.1: $x=2$

Since $v \geq 4$ we have $\left|V_{2}\right|>1$ and $y>0$. Let $v_{2} \in V_{2}$ and $w_{1}$ and $w_{2}$ be distinct vertices in $W_{1}$. Partition the vertices in $V_{2} \backslash\left\{v_{2}\right\}$ into pairs, say $P_{1}, P_{2}, \ldots, P_{(v-2) / 2}$. For each $i \in\{1,2, \ldots,(v-2) / 2\}$ we let $H_{i}$ be the complete bipartite graph with partite sets $\left\{v_{1}, w_{1}, w_{2}\right\}$ and $P_{i}$. It is clear that each $H_{i}$ can be decomposed into two non-stars of size 3 . If $\alpha \geq v-2$, we let $H=\bigcup_{i=1}^{(v-2) / 2} H_{i}$ and thus $G=K_{a_{1}, a_{2}, \ldots, a_{n}} \backslash H$ has an (open) Euler trail. Similarly, if $\alpha<v-2$ and $\alpha$ is even, we let $H=\bigcup_{i=1}^{\alpha / 2} H_{i}$ and hence $G=K_{a_{1}, a_{2}, \ldots, a_{n}} \backslash H$ satisfies the conditions of Lemma 2.3 , with $G^{\prime}$ the star having center vertex $v_{1}$ and boundary $\bigcup_{i=\alpha / 2+1}^{(v-2) / 2} P_{i}$. Finally, if $\alpha<v-2$ and $\alpha$ is odd, we let $H=\left(\bigcup_{i=1}^{(\alpha-1) / 2} H_{i}\right) \cup\left[w_{1}, v_{1}, v_{2}, w_{2}\right]$. Then $G=K_{a_{1}, a_{2}, \ldots, a_{n}} \backslash H$ satisfies the conditions of Lemma 2.3, with $G^{\prime}$ the star having center vertex $v_{1}$ and boundary $\left(\bigcup_{i=(\alpha+1) / 2}^{(v-2) / 2} P_{i}\right) \cup\left\{w_{2}\right\}$.

Subcase 2.2.2: $x \geq 4$

Let $w_{1} \in V_{x-2}, w_{2} \in V_{x-1}$ and $w_{3} \in V_{x}$. Partition the vertices in $\bigcup_{i=2}^{x-1} V_{i}$ into pairs, say $P_{0}=\left\{w_{1}, w_{2}\right\}, P_{1}, \ldots, P_{r}$, and if $\left|V_{x}\right|>1$ (that is, $r<(v-4) / 2$ ), partition the vertices in $V_{x} \backslash\left\{w_{3}\right\}$ into pairs, say $P_{r+1}, P_{r+2}, \ldots, P_{(v-4) / 2}=\left\{u_{1}, u_{2}\right\}$. If $0<r<(v-4) / 2$, then for each $i \in\{1,2, \ldots, r\}$ we let $H_{i}$ be the complete bipartite graph with partite sets $\left\{v_{1}, u_{1}, u_{2}\right\}$ and $P_{i}$, and for each $i \in\{r+1, r+2, \ldots,(v-4) / 2\}$ we let $H_{i}$ be the complete bipartite graph with partite sets $\left\{v_{1}, w_{1}, w_{2}\right\}$ and $P_{i}$. If $r=(v-4) / 2>0$ or $r=0$, then for each $i \in\{1,2, \ldots,(v-4) / 2\}$ we let $H_{i}$ be the complete bipartite graph with partite sets $\left\{v_{1}, w_{1}, w_{2}\right\}$ and $P_{i}$. It is clear that each $H_{i}$ can be decomposed into two non-stars of size 3 . If $\alpha \geq v-3$, we let $H=\left(\bigcup_{i=1}^{(v-4) / 2} H_{i}\right) \cup\left[v_{1}, w_{1}, w_{2}, w_{3}\right]$ otherwise. Then $G=K_{a_{1}, a_{2}, \ldots, a_{n}} \backslash H$ has an (open) Euler trail. Similarly, if $\alpha<v-3$ and $\alpha$ is even, we let $H=\bigcup_{i=1}^{\alpha / 2} H_{i}$ and hence $G=K_{a_{1}, a_{2}, \ldots, a_{n}} \backslash H$ satisfies the conditions of Lemma 2.3, with $G^{\prime}$ the star having center vertex $v_{1}$ and boundary $\left(\bigcup_{i=\alpha / 2+1}^{(v-4) / 2} P_{i}\right) \cup\left\{w_{1}, w_{2}, w_{3}\right\}$. Finally, if $\alpha<v-3$ and $\alpha$ is odd, we let $H=\left(\bigcup_{i=1}^{(\alpha-1) / 2} H_{i}\right) \cup\left[v_{1}, w_{1}, w_{2}, w_{3}\right]$. Then $G=K_{a_{1}, a_{2}, \ldots, a_{n}} \backslash H$ satisfies the conditions of Lemma 2.3, with $G^{\prime}$ the star having center vertex $v_{1}$ and boundary $\bigcup_{i=(\alpha+1) / 2}^{(v-4) / 2} P_{i}$. 


\section{Main Result}

We are now ready to prove our main result.

Proof of Theorem 1.1 Let $G=K_{2 a_{1}, 2 a_{2}, \ldots, 2 a_{n}}$, for $n \geq 2$. If $n=2$ and $\left\{a_{1}, a_{2}\right\}=\{1, a\}$ for some $a \geq 1$, then it is easy to see that $\operatorname{Lcet}(G)=\{4,8, \ldots, 4 a\}$ : since $G=K_{2,2 a}$ has a decomposition into closed trails of length 4 , each of which intersects both vertices in the partite set of size 2, a decomposition of $G$ into closed trails whose lengths belong to Lcet $(G)$ is easily obtained.

Suppose then that either $n \geq 3$, or $n=2$ and $\left\{a_{1}, a_{2}\right\} \neq\{1, a\}$ for each $a \geq 1$. Thus $K_{a_{1}, a_{2}, \ldots, a_{n}}$ is ADNS by Theorem 2.4. Since $G \cong K_{a_{1}, a_{2}, \ldots, a_{n}}(2)$ the result then follows by Lemma 2.2.

\section{References}

[1] P. Balister, Packing circuits into $K_{N}$, Combin. Probab. Comput. 10 (2001), 463-499.

[2] E. J. Billington, N. J. Cavenagh, Decomposing complete tripartite graphs into closed trails of arbitrary lengths, Czechoslovak Math. J. 57 (2007), 523-551.

[3] A. Burgess, M. Šajna, Closed trail decompositions of complete equipartite graphs, J. Combin. Designs 17 (2009), 374-403.

[4] M. Horňák, M. Woźniak, Decomposition of complete bipartite even graphs into closed trails, Czechoslovak Math. J. 53 (2003), 127-134.

[5] B. R. Smith, Decomposing complete equipartite graphs into closed trails of length $k$, Graphs Combin. 26 (2010), 133-140. 